\title{
Gender-related differences in neuroscience - gender- and sex-related diseases
}

\author{
Hilmar Walter Prange \\ Department of Neurology, University Hospital, Georg August University of Göttingen, Germany
}

\begin{abstract}
Whether men and women are fundamentally different or similar has been debated for many years. The incidence, age of onset and symptomatology of many diseases shows the differences between men and women. In health, more than in other social sectors, sex and gender variables are useful parameters for research and action because biological differences between the sexes determine male-specific and female-specific diseases and because behavioral differences between the genders assign a critical role to women in relation to family health. This article highlights some of the more intriguing recent opinion and their consequences in the subject of gender-related diseases.
\end{abstract}

KEY WORDS: sex, neuroscience, gender differences, sex-related diseases.

ADDRESS FOR CORRESPONDENCE: Prof. Hilmar Walter Prange, Department of Neurology, University Hospital, Georg August University of Göttingen, Robert Koch Str. 40, D-37075 Göttingen, Germany, phone: 004955121170 , fax: 004955139 8405, e-mail: hilmarprange@gmx.de

\section{INTRODUCTION}

Both sex and gender are interactive components in health. The term sex is used to describe differences between men and women that are primarily biological in origin and that may be genetic or phenotypic. The concept of gender aims to describe differences that are primarily a result of social conditions or cultural and religious attitudes as well as norms regarding the sexes. Structural gender inequalities placing women in a subordinate position to men contribute to gender differentials in disease [1]. The corresponding approach to the role of gender should address the differences between men's health and women's health that result from the different positions in the respective society. Sex and gender can act alone, independently, or interactively in determining differentials in the burden of disease [2].

Additionally, in health, more than in other social sectors, sex and gender variables are useful parameters for research and action because biological differences between the sexes determine male-specific and femalespecific diseases and because behavioral differences between the genders assign a critical role to women in relation to family health.

\section{BASAL BIOLOGICAL ASPECTS}

Even though the brain size is greater in men than in women, the ratio of brain mass to body mass is higher in female people, according to $1: 46(22 \mathrm{~g}$ per $1 \mathrm{~kg})$ in women vs. $1: 50(20 \mathrm{~g}$ per $1 \mathrm{~kg})$ in men. Irrespective of this fact, size of the brain does not really say anything about its performance. Interestingly, the sex differences of the average size are already present in newborns. They persist until the adult age. The "intrinsic blueprint" of brain is female, but early in the embryonic stage an $\mathrm{X}$ - and Y-chromosome-dependent respectively hormone-dependent differentiation is occuring. The actually active hormone is estradiol which is converted within the brain into testosterone. This reaction does not happen in female brains due to the presence of a special $\alpha$-fetoprotein. Even in the adult brains there are morphological differences between men and women. Circumscribed damages of special cortical or subcortical areas can sex-dependently result in different functional impairments $[3,4]$. This applies e.g. to aspects of aphasia and apraxia. The dexterity is regularly less pronounced in females which could be an advantage for women in learning languages. 
The maturation of cognitive capabilities of the brain depends on the interaction with the environment and is eventually based on learning processes during the individual development. Thus, there are differences in problem-solving strategies between the two sexes. This concerns inter alia spatial imagination, mathematical deductions and speed of perception. These functions seem to be dependent on testosterone.

For instance, females with higher testosterone levels show a better performance in spatial imagination than those with lower levels, however there is a reverse effect in males [3].

Regarding pathological conditions it is known that hormonal influences can promote or mitigate the manifestation of a disease. With women, for example, high levels of estrogen seem to protect against schizophrenia or at least are reducing the symptoms [5]. On the other hand, it was proposed that reduced hippocampal volume and impaired hypothalamus-pituitary-adrenal axis demonstrated by an attenuated cortisol awakening response are related markers of increased stress vulnerability in male psychosis patients and contribute to the unfavorable clinical picture [6]. Apart from that, the prevalence of schizophrenia amounts to $0.5-1.0$ per 100 inhabitants. There are no significant differences between the sexes. But with so-called disorganized schizophrenia (synonym: hebephrenia) the manifestation occurs earlier in male than in female patients, $90 \%$ before the age 30 in males versus $66 \%$ in females. Yet the prevalence of this subtype of schizophrenia is significantly higher in females. Sex-related rather than gender-related factors may play a role in this case [7].

\section{HEALTH-RELATED CONSIDERATIONS}

In the past, medical research often wrongly assumed that women were biologically weaker (male bias) and extrapolated findings from trials with male subjects only (male norm) to both sexes, whereas female biology can affect the onset and progression of disease, and women's lower position in many societies can affect their health-seeking behaviors. Of special interest is using global burden-of-disease data and gender differentials in mortality and morbidity that result in excess disease burdens for women. The focus on women's excess disease burden is justified to fill gaps in knowledge regarding women's health that are in part a product of male bias and male norms in clinical studies [1,8]. Thus, the burden of disease (BOD) is a useful instrument for assessing differences in health condition between men and women. It is based on disability-adjusted life years (DALYs)

As mentioned above, in most cases sex and gender interact to determine women's disease burdens. A salient example is the depression. Women are twice as likely as men to become depressed, and genetics and hormones influence the risk of depression.
However, genes and sex hormones cannot entirely explain women's excess burdens, and gender factors may play an important role. Existing knowledge about the interplay between sex and gender in determining disease is imperfect but growing $[2,8]$. There are several possibilities to categorize women's excess health burdens from diseases as follows:

- diseases specific to women (biology plays a major role in the disease) e.g. complications of eclampsia or breast cancer,

- diseases related to women's average greater longevity (where both sex and gender tend to play important roles) e.g. Parkinson's disease or stroke,

- diseases that result from the interaction of sex and gender, e.g. unipolar depression,

- diseases which are predominantly gender based (resulting from specific behavioral, social, and cultural factors associated with women's condition), e.g. sexual mutilations in many countries.

Sex and gender have a much wider influence on disease than is usually acknowledged. They influence the etiology, diagnosis, progression, prevention, treatment, and health outcomes of disease as well as health-seeking behaviors and exposure to risk. Whereas sex plays a bigger role in the etiology, onset, and progression of disease, gender and its consequences influence differential risks, symptom recognition, severity of disease, access to and quality of care, and compliance with care. Again, factors that influence gender differentials in relation to the risk of disease include (a) biological differences between the sexes; (b) women's longer life expectancy; (c) women's labor force participation compared with men's participation; (d) women's access to social protection mechanisms; (e) cultural norms, religious beliefs, and gender hierarchy in family and society; (f) gender differences in educational attainment; (g) income differences between the genders. Women's overall underutilization of health services has been documented for many countries $[9,10]$. In the developed countries, the number of women age 80 and older is more than double the number of men in the same age group. Among other factors, this female advantage in longevity results is a bias in gender ratio (on the base of DALY) and BOD as shown in Table 1.

The main groups of diseases with excess burdens for women associated with women's greater longevity are Alzheimer's disease, musculoskeletal disorders, such as osteoarthritis, rheumatoid arthritis, and osteoporosis, and cardiovascular diseases, which together account for $12 \%$ of total DALY losses for women worldwide [12]. Alzheimer's disease and other dementias account for $1.5 \%$ of total female DALYs, and this burden is almost twice as high as that for men. Osteoarthritis impairs $9.6 \%$ of men and $18.0 \%$ of women age 60 or older worldwide and accounts for $1.5 \%$ of total female DALYs. Osteoarthritis is related to aging and is most common 
TABLE 1. Gender ratio and women's excess burden of disease for top priority conditions, 2001 [11]

\begin{tabular}{|l|c|c|c|c|c|c|}
\hline \multirow{2}{*}{ Condition } & \multicolumn{2}{|c|}{ World } & \multicolumn{2}{c|}{ LMIC } & \multicolumn{2}{c|}{ HIC } \\
\cline { 2 - 7 } & GR & BOD (\%) & GR & BOD (\%) & GR & BOD (\%) \\
\hline Alzheimer's disease and other dementias & 1.81 & 1.48 & n.a. & n.a. & 2.13 & 7.02 \\
\hline Osteoarthritis & 1.64 & 1.46 & 1.63 & 1.26 & 1.73 & 3.30 \\
\hline Unipolar depressive disorders & 1.53 & 4.22 & 1.51 & 3.89 & 1.69 & 7.30 \\
\hline Age-related vision disorders (Europe) & 1.63 & 2.10 & n.a. & n.a. & n.a. & n.a. \\
\hline Migraine (Latin America) & 2.81 & 1.14 & n.a. & n.a. & n.a. & n.a. \\
\hline Cerebrovascular diseases (sub-saharan Africa) & 1.47 & 1.80 & n.a. & n.a. & n.a. & n.a. \\
\hline
\end{tabular}

Source: WHO 2001 (see [11]). n.a. - not applicable; BOD - burden of disease; GR - gender ratio which is ratio of female to male DALYs; LMIC - low and moderate income countries; HIC - high income countries (methodology used in this study: the gender ratio was equal to or higher than the mean plus one SD of the distribution of gender ratio scores, the BOD was equal to or higher than the mean plus one SD of the distribution of female DALY scores)

in overweight women over the age of 45 . Cardiovascular and cerebrovascular diseases account for about $8.2 \%$ of total female DALYs, more than half of which is caused by cerebrovascular diseases. In addition to age, smoking, and obesity, another risk factor that exposes women to a greater burden of cardiovascular diseases is depression, which is associated with increased morbidity and mortality from heart diseases and is highly prevalent in women [13].

Unipolar depressive disorders have the most significant gender ratio and most unequal global BOD. They account for $4.2 \%$ of women's BOD. Even though the DALYs lost per capita are similar in low and moderate income countries (LMICs) and high income countries (HICs), these disorders represent a higher share of women's total BOD of disease in HICs (7.3\%) than in LMICs (3.9\%).

\section{BURDEN OF DISEASE IN THE MOST FREQUENT NEUROLOGICAL DISEASES}

According to World Health Organization (WHO), neurological and psychiatric disorders are a growing cause of morbidity. Mental, neurological, and behavioral disorders affect more than 450 million people globally. Taking the Global Burden of Disease Report of WHO, $33 \%$ of years lived with disability and $13 \%$ of DALYs are due to neurological and psychiatric disorders [14]. Patients who are suffering from these conditions are often victims of stigmatization and discrimination, which limits patient's access to therapy.
The prevalence of dementia has generally been found to double with every five-year increase in age, from 3/100 at age 70 to $20-30 / 100$ at age 85 . The genes APP, PS1, and PS2 are connected with early-onset familial Alzheimer's dementia. Apolipoprotein E4 plays a role for the manifestation of non-familial cases. Risk factors are increasing age, positive family history of dementia, female gender, lower level of education, several medical conditions, and exposure to organic solvents and aluminum. Factors assumed as protective are higher level of education, intake of anti-oxidants, and use of some anti-inflammatory medications [15]. Estrogen supplements for women could be a protective factor for Alzheimer's dementia but the studies to this subject are dissenting [16, 17]. Burden of disease estimates of Alzheimer's dementia and other dementias include vascular dementia, unspecified dementias, and other unclassified degenerative diseases of the nervous system. Mathers et al. [14] calculated DALYs for all dementias with the result of $17,108,000$, with the burden being almost twice as much for females $(11,016,000)$ as for males $(6,092,000)$.

Epilepsy is a common brain disorder characterized by two or more unprovoked seizures. The prevalence of active epilepsy globally is in the range of 5 to 8 per 1,000 inhabitants [18]. A reported risk factor for idiopathic (presumed genetic) epilepsy is family history of epilepsy. Reported risk factors for symptomatic epilepsy include prenatal or perinatal causes, traumatic brain injuries (TBI), central nervous system infections, cerebrovas-

TABLE 2. Disability-adjusted life years (DALY) in thousands (restricted to global data, 2001) [11]

\begin{tabular}{|l|c|c|c|}
\hline Condition & Both sexes & Females & Males \\
\hline Alzheimer's disease and other dementias & 17,108 & 11,016 & 6,092 \\
\hline Epilepsy & 6,223 & 2,922 & 3,301 \\
\hline Parkinson's disease & 2,325 & 1,202 & 1,124 \\
\hline Cerebrovascular diseases & 72,024 & 36,542 & 35,482 \\
\hline
\end{tabular}


cular disease, brain tumors, and neurodegenerative diseases [19, 20]. Mathers et al. [14] estimate the DALYs for epilepsy as $6,223,000$, with slightly higher rates for males $(3,301,000)$ than for females $(2,922,000)$. Since the incidence of TBI is much higher in men (86\%) than in women (16\%) it is not surprising that DALY is slightly predominating in males.

Parkinson's disease is characterized by bradykinesia, resting tremor, cogwheel rigidity, postural reflex impairment, progressive course, and good response to dopaminergic therapy. Genetic forms are relatively rare (10-15\%). Van den Eeden et al. reported the incidence rate of Parkinson's disease in the United States as approximately 13 per 100,000 person-years [21]. Men are affected more commonly than women [22]. Increasing age and male gender are risk factors worldwide [23]. The most consistent association is an inverse association with caffeine consumption, suggesting a protective effect [24]. Mathers et al. [14] estimate the DALYs for Parkinson's disease as 2,325,000, with the burden being slightly higher in females $(1,202,000)$ than males $(1,124,000)$. Even though male gender is a risk factor for Parkinson's disease, the higher burden in females may reflect their longer life span.

Cerebrovascular accidents are in more than $75 \%$ ischemic strokes, caused by an occlusion of a cerebral blood vessel. The rupture of a cerebral blood vessel results in a hemorrhagic stroke (about $20 \%$ of all strokes). According to the DEGAM guideline [25] annual incidence of the stroke amounts in Germany to 1.82 per 1000 inhabitants with predominance of the men (women $1.7 / 1000$, men 2.0/1000). Although stroke is more prevalent among men, stroke-related fatality rates are higher among women. Taken the global incidence and prevalence, there is a predominance of females among the afflicted individuals, e.g. the male-to-female ratio was in India according Anand et al. (2001) one to seven [26]. Risk factors for stroke are increasing age, particularly after 55, smoking, arterial hypertension, dyslipidemia, atrial fibrillation, diabetes, generalized and abdominal obesity, physical inactivity, increased homocysteine levels, drug and alcohol abuse, hemostatic factors, and existing cerebrovascular disease. Preclinical studies indicate that steroid hormones modulate the age-dependent differential stroke outcome. In addition, patterns of cell death pathways activated following cerebral ischemia are distinct between males and females [27].

The BOD estimates for stroke include subarachnoid hemorrhage, intracerebral hemorrhage, cerebral infarction, and sequelae of cerebrovascular disease [14]. They are almost similar for females $(36,542,000)$ and males $(35,482,000)$.

\section{NEUROLOGICAL DISORDERS WITH DIFFERENT SEX/GENDER DISTRIBUTION}

The vast majority of hereditary diseases in neurology does not show any differences in sex distribution unless the gene is located on the X- or Y-chromosome. The opposite is the case in conditions with hormonal influences. In both conditions the gender aspect does not play a special role in the first instance. As already mentioned above, in many other diseases the influence of biological factors (sex) and social factors (gender) is hard to untangle because the factors are mutually dependent. It is therefore essential to record the diseases with different sex distribution exploring the circumstances that may influence the evolving process. This is of therapeutic, prophylactic and epidemiological relevance and can be useful to recognize hidden pathogenic mechanisms. It is conceivable that sociological laws are becoming obvious on this way.

In the following we are presenting a compilation of neurological diseases with known differences of sex distribution which mainly derives from German disease networks and guidelines. Previously, in Table 3 there are shown the sex differences with patients visiting special out-patient clinics for dementia in Germany. One can recognize that the portion of men is higher than that of women. The sex distribution in Alzheimer's disease shows a slightly higher rate for women which, however, is not reflecting the gender rate with significant female predominance reported from many other countries. It can be assumed that costumary and/or social restraining mechanisms are preventing some females to visit special out-patient clinics.

The compilation of Table 4 shows differences in the sex distribution for several neurological conditions. The reasons for these inequalities are in the most cases not recognizable at the first glance. Especially, there is often the role of biological (sex) and social (gender) aspects not to distinguish. In diseases with a neuroimmunological pathogeneses like myasthenia gravis, multiple sclerosis [28] or cranial arteriitis there is in the most cases a higher incidence with women. The social role in pathogenesis is at this undefined and there are deviations from the rule as shown with Guillain-Barré syndrome and inclusion body myositis.

In the course of the diseases with neuroimmunological origin, social, occupational and costumary factors may modify symptoms and outcome. Influence of hormones and other biochemical circumstances seems to be evident with the majority of brain tumors [29]. Gender-related factors should be less effective than the biological ones. In diseases manifesting with pain, women display the higher incidence. Typical instances are migraine, trigeminal neuralgia, atypical facial pain, benign paroxysmal vertigo, and complex regional pain syndromes (CRPS). All of these conditions are organic in origin which is well defined, but social and environmental factors may unfold a remarkable influence with the symptom development. Occupational influences may be stronger in conditions like carpal tunnel syndrome, ulnar neuropathy and amyotrophic lateral sclerosis (ALS). In spite of that, an anatomic proneness should not be overlooked in the entrapment syndromes. The male predominance in meningitis and traumatic brain injury 
TABLE 3. Sex distribution in an out-patient clinic for dementia in Munich, Germany [17]

\begin{tabular}{|l|c|c|c|}
\hline & Male patients $(n)$ & Female patients $(n)$ & Total $(n)$ \\
\hline Alzheimer's disease & 82 & 97 & 179 \\
\hline Frontotemporal degeneration & 21 & 15 & 15 \\
\hline Vascular dementia & 12 & 1 & 6 \\
\hline Dementia by alcohol abuse & 5 & 1 & 8 \\
\hline Dementia with other diseases & 7 & 5 & 17 \\
\hline Unclear dementia & 12 & 122 & 261 \\
\hline Total & 139 & 3 & 17 \\
\hline
\end{tabular}

TABLE 4. Neurological diseases with unequal sex distribution

\begin{tabular}{|c|c|c|c|}
\hline Condition & Incidence rate & Prevalence & Femal-to-male ratio \\
\hline Brain tumors (total) & $7-10 / 100,000$ & & $1: 1.35$ \\
\hline Glioblastoma & $2.9-3.5 / 100,000$ & & $1: 2$ \\
\hline Pinealoma & $0.02-0.08 / 100,000$ & & $1: 12$ \\
\hline Pituitary tumors & $6-8 / 100,000$ & & \\
\hline Inactive adenomas & $1 / 100,000$ & & $1: 2.4$ \\
\hline Prolactinoma & $2-5 / 100,000$ & & $2.5: 1$ \\
\hline Migraine & & $6-8 / 100$ & $2: 1$ \\
\hline Trigeminal neuralgia & $3.9-5.9 / 100,000$ & & $1.5: 1$ \\
\hline Cluster headache & & $1-9 / 1000$ & $1: 3$ \\
\hline Atypical facial pain & $4.4 / 100,000$ & & $8.2: 1$ \\
\hline Benign paroxysmal vertigo & & $2.4 / 100$ & $2: 1$ \\
\hline Complex regional pain syndrome (CRPS) & $6-26 / 100,000$ & & $3: 1$ \\
\hline Carpal tunnel syndrome & $3,5 / 1000$ & & $6: 1$ \\
\hline Ulnar neuropathy (SUS) & $25 / 100,000$ & & $1: 2$ \\
\hline Guillain-Barré syndrome & $1.2-2.3 / 100,000$ & & $1: 1.5$ \\
\hline Myasthenia gravis & & $4-9 / 10,000$ & \\
\hline Until age 20 & & & $6.7: 1$ \\
\hline Age $>60$ & & & $1: 1.7$ \\
\hline Inclusion body myositis (spontaneous variant) & $0.5 / 100,000$ & & $1: 3$ \\
\hline Amyotrophic lateral sclerosis (ALS) & $1.5-2 / 100,000$ & & $1: 1.5$ \\
\hline Multiple sclerosis & $5-6 / 100,000$ & & $2: 1$ \\
\hline Cranial arteriitis (age $>50$ ) & $8.2-24.2 / 100,000$ & & $3: 1$ \\
\hline Spontaneous subarachnoid hemorrhage (SAH) & $10.5 / 100,000$ & & $1.6: 1$ \\
\hline Multiple system atrophy (MSA) age $>50$ & $3 / 100,000$ & & $1: 1.4$ \\
\hline Narcolepsy & $0.74 / 100,000$ & & $1: 1.2$ \\
\hline Bacterial meningitis (Germany) & 3/100,000 (Europe) & & $1: 2$ \\
\hline Traumatic brain injury (TBI) (Germany) & $332 / 100,000$ & & $1: 5.4$ \\
\hline
\end{tabular}

Sources: German networks and guidelines of the German Neurological Society (DGN). 
may be a consequence of higher exposition and reduced perception of jeopardy. This is a typical gender-related feature. The higher incidence of subarachnoid hemorrhage $(\mathrm{SAH})$ in females could probably been explained by relatively weaker connective tissue in women [30].

In summary it can be confirmed that in some diseases biological and in others social factors play a greater role in shaping symptoms and course. That there are many additional factors deriving from environment, family, and occupation was mentioned above, not to forget the interactive mechanisms between patient and environment. Literature concerning with this subject contains comparatively little about which diseases affect men and women differently, why that difference might be the case, and how to structure prevention and treatment in response to these differences. This situation is changing, however, and interest in measuring, understanding, and responding to sex and gender differentials in disease has been increasingly encouraged in the recent years. One can expect conclusive concepts for the future.

\section{DISCLOSURE}

Author reports no conflict of interest.

\section{References}

1. Sen G, George A, Östlin P. Engendering health equity: a review of research and policy. In: Engendering International Health. Sen G, George A, Östlin P (eds.). Massachusetts Institute of Technology Press, Cambridge 2002; 1-33.

2. Krieger N. Gender, sexes, and health: what are the connections and why does it matter? Int J Epidemiol 2003; 32: 652-657.

3. Kimura D, Harshman RA. Sex differences in brain organization for verbal and nonverbal function. In: Sex differences in the brain. De Vries GJ, DeBruin JP, Uylings HB, Corner MA (eds.). Elsevier, Amsterdam 1984; 423-439.

4. Springer SP, Deutsch G. Linkes-rechtes Gehirn: Funktionelle Asymmetrien. Spektrum der Wissenschaft, Heidelberg 1987.

5. Riecher-Rössler A. Östrogene und schizophrene Psychosen. In: Sexualhormone und Psyche: Grundlagen, Symptomatik, Erkrankungen, Therapie. Kuhl H (ed.). Verlag Thieme, Stuttgart 2002; S38-47.

6. Pruessner M, Lepage M, Collins DL, et al. Reduced hippocampal volume and hypothalamus-pituitary-adrenal axis function in first episode psychosis: evidence for sex differences. Neuroimage Clin 2014; 7: 195-202.

7. Vyas NS, Hadjulis M, Vourdas A, et al. The Maudsley early onset schizophrenia study: Predictors of psychosocial outcome at 4-years follow-up. Eur Child Adolesc Psychiatry 2007; 16: 465-470.

8. Pinn VW. Sex and gender factors in medical studies. Implications for health and clinical practice. JAMA 2003; 289: 397-400.

9. Levine R, Glassman A, Schneidman M. La Salud de la Mujer en América Latina y el Caribe. Inter-American Development Bank, Washington, DC 2001.

10. Hanson K. Measuring up: gender, burden of disease, and priority setting. In: Engendering International Health. Sen G, George A, Östlin P (eds.). Massachusetts Institute of Technology Press, Cambridge, MA; 2002.
11. Jamison DT, Breman JG, Measham AR, et al. (eds.). Disease control priorities in developing countries. $2^{\text {nd }} \mathrm{ed}$. World Bank, Washington 2006.

12. Buvinić M, Medici A, Fernández E, Torres AC. Gender differentials in health. In: Disease control priorities in developing countries. $2^{\text {nd }}$ ed. Jamison DT, Breman JG, Measham AR, et al. (eds.). World Bank, Washington 2006.

13. Linfante AH, Allan R, Smith SC, Mosca L. Psychosocial factors predict coronary heart disease, but what predicts psychosocial risk in women? J Am Med Womens Assoc 2003; 58: 248-253.

14. Mathers CD, Lopez A, Stein C, et al. Deaths and Disease Burden by Cause: Global Burden of Disease Estimates for 2001 by World Bank Country Groups. World Bank, Washington 2003.

15. Henderson AS, Jorm AS. Definition of epidemiology of dementia: a review. In: Dementia. Mario M, Sartorius N (eds.). John Wiley, West Sussex 2000; 1-34.

16. Henderson VW. The epidemiology of estrogen replacement therapy and Alzheimer's disease. Neurology 1997; 48 (5 Suppl. 7): S27-35.

17. Bickel H, Bürger K, Hampel H, et al. Presenile dementia in memory clinics - incidence rates and clinical features. Nervenarzt 2006; 77: 1079-1085.

18. Leonardi M, Ustun TB. The global burden of epilepsy. Epilepsia 2002; 43 (Suppl. 6): 21-25.

19. Casetta I, Monetti VC, Malagu S, et al. Risk factors for cryptogenic and idiopathic partial epilepsy: A community-based case-control study in Copparo, Italy. Neuroepidemiology 2002; 21: 251-254

20. Leone M, Bottacchi E, Beghi E, et al. Risk factors for a first generalized tonic-clonic seizure in adult life. Neurological Sciences 2002; 23: 99-106.

21. Van Den Eeden SK, Tanner CM, Bernstein AL, et al. Incidence of Parkinson's disease: Variation by age, gender, and race/ethnicity. Am J Epidemiol 2003; 157: 1015-1022.

22. Tanner C, Goldman S. Epidemiology of Parkinson's disease. Neurol Clin 1996; 14: 317-335.

23. Marras C, Tanner C. The epidemiology of Parkinson's disease. In: Movement disorders neurologic principles and practice. Watts RL, Koller WC (eds.). McGraw-Hill, New York 2002; 177-196.

24. Ascherio A, Zhang SM, Hernan MA, et al. Prospective study of caffeine consumption and risk of Parkinson's disease in men and women. Ann Neurol 2001; 50: 56-63.

25. DEGAM: S3 Guideline Stroke (Schlaganfall). www.degam.de/ leitlinien.html

26. Anand K, Chowdhury D, Singh KB, et al. Estimation of mortality and morbidity due to stroke in India. Neuroepidemiology 2001; 20: 208-211.

27. Kim TH, Vemuganti R. Effect of sex and age interactions on functional outcome after stroke. CNS Neurosci Ther 2015; 21: 327-336.

28. Voskuhl RR. Gender issues and multiple sclerosis. Curr Neurol Neurosci Rep 2002; 2: 277-286.

29. Mindermann T, Wilson CB. Age-related and gender-related occurrence of pituitary adenomas. Clin Endocrinol (Oxf) 1994; 41: 359-364.

30. Kongable GL, Lanzino G, Germanson TP, et al. Gender-related differences in aneurysmal subarachnoid hemorrhage. J Neurosurg 1996; 84: 43-44. 